\title{
Review of: "Linear unmixing protocol for hyperspectral image fusion analysis applied to a case study of vegetal tissues"
}

\author{
Douglas Barbin ${ }^{1}$ \\ 1 Universidade Estadual de Campinas
}

Potential competing interests: The author(s) declared that no potential competing interests exist.

The paper reports a practical case-study on the application of MCR-ALS to evaluate each of the components of a biological sample (rice leaves) that contribute to the spectral signal in hyperspectral images. The main novelty resides on the application of MCR-ALS to deal with the combination of SR-FTIR, Raman and fluorescence HSI from rice leaf cross-sections.

MCR-ALS has previously been reported for several applications on HSI, to deal with different components within a sample such as food, plant cells, etc.

- Mahdiyeh Ghaffari, Nematollah Omidikia, Cyril Ruckebusch. (2019). Essential Spectral Pixels for Multivariate Curve Resolution of Chemical Images. Anal. Chem., vol. 91 (17), 10943-10948. doi:10.1021/acs.analchem.9b02890 [1]

- Amanda Teixeira Badaró, José Manuel Amigo, Jose Blasco, Nuria Aleixos, et al. (2021). Near infrared hyperspectral imaging and spectral unmixing methods for evaluation of fiber distribution in enriched pasta. Food Chemistry, vol. 343 , 128517. doi:10.1016/j.foodchem.2020.128517.[2]

- Batirtze Prats-Mateu, Martin Felhofer, Anna de Juan, Notburga Gierlinger. (2018). Multivariate unmixing approaches on Raman images of plant cell walls: new insights or overinterpretation of results?. Plant Methods, vol. 14 (1). doi:10.1186/s13007-018-0320-9.[3]

However, this was the first time that SRFTIR, Raman and fluorescence HSI are fused and analysed by MCRALS to profoundly investigate the distinctive constituents and structures that are part of the tissues of plant leaves. As stated by the authors, this approach required that "images from the different platforms had to be balanced in terms of spatial resolution, orientation and area scanned before being analysed." The authors present an explanation of the challenges of fusing images from such different equipment, in section "Data analysis - HSI preprocessing". Also, the approach described in 'Building a multiset with congruent pixels" demonstrate the challenge of the proposed method.

The approach is clearly described and the results are sound and corroborate the potential that MCR-ALS can provide for such applications. There will be future demands from micro and macro analysis that will benefit from the proposed approach, including several types of analysis where different imaging systems may complement each other, providing a deeper understanding of components in biological samples. 


\section{References}

1. ^Mahdiyeh Ghaffari, Nematollah Omidikia, Cyril Ruckebusch. (2019). Essential Spectral Pixels for Multivariate Curve Resolution of Chemical Images. Anal. Chem., vol. 91 (17), 10943-10948. doi:10.1021/acs.analchem.9b02890.

2. A Amanda Teixeira Badaró, José Manuel Amigo, Jose Blasco, Nuria Aleixos, et al. (2021). Near infrared hyperspectral imaging and spectral unmixing methods for evaluation of fiber distribution in enriched pasta. Food Chemistry, vol. 343 , 128517. doi:10.1016/j.foodchem.2020.128517.

3. `Batirtze Prats-Mateu, Martin Felhofer, Anna de Juan, Notburga Gierlinger. (2018). Multivariate unmixing approaches on Raman images of plant cell walls: new insights or overinterpretation of results?. Plant Methods, vol. 14 (1). doi:10.1186/s13007-018-0320-9. 